\title{
Effect of Diet on the Development of Drug Metabolism by Cytochrome P-450 Enzymes in Healthy Infants
}

\author{
MICHAEL J. BLAKE, SUSAN M. ABDEL-RAHMAN, ROBIN E. PEARCE, J. STEVEN LEEDER, AND GREGORY L. KEARNS \\ Department of Pediatrics, University of Missouri-Kansas City, School of Medicine and the Division of Pediatric Pharmacology \\ and Medical Toxicology, Children's Mercy Hospitals and Clinics, Kansas City, Missouri 64108
}

\begin{abstract}
Orally administered caffeine and dextromethorphan (DM) were used as pharmacologic probes to determine the effect of infant diet on acquisition of cytochrome P-450 (CYP) enzyme activity during the first 6 mo of life. The caffeine elimination rate constant $\left(\mathrm{k}_{\mathrm{e}}\right)$ was determined from serum, and concentrations of caffeine, DM, and their respective metabolites were measured in urine by highperformance liquid chromatography (HPLC). Caffeine $\mathrm{k}_{\mathrm{e}}$ was low at $2 \mathrm{wk}$ and displayed a significant positive linear correlation with age $(p<0.001)$; increasing faster in formula-fed than in breast-fed infants $(p<0.001)$. This occurred concomitantly with a significant increase in urinary 1,7-dimethylxanthine (17X) and 1-methylxanthine $(1 \mathrm{X})(p<0.001)$, suggesting faster acquisition of CYP1A2 activity in formula-fed infants. The urinary molar ratio of $(17 \mathrm{X}+$ $1 \mathrm{X}) /$ caffeine and age strongly predicted caffeine $\mathrm{k}_{\mathrm{e}}\left(r^{2}=0.65 ; p<\right.$ 0.001 ) irrespective of feeding type. CYP3A4 activity, assessed as the molar ratio of 3-hydroxymorphinan/dextrorphan showed a similar marked increase with postnatal age $(p<0.001)$ that was also greater in formula-fed than in breast-fed infants. Formula feeding appears to accelerate maturation of caffeine and DM metabolism by increasing the activity of CYP1A2 and CYP3A4, respectively. Dietary modification of CYP activity may modulate drug biotransformation and thus alter systemic exposure to xenobiotics from a very early age. (Pediatr Res 60: 717-723, 2006)
\end{abstract}

$\mathrm{T}$ he benefits of breast-feeding have been recognized for centuries. Only more recently have the bases of these advantages begun to be elucidated. The identification of specific immunoglobulins in breast milk and the influence of breast milk on gastrointestinal function and intestinal flora are notable examples (1). Emerging data now support a positive influence of breast-feeding on many facets of child health and development (2-4). An aspect of breast-feeding that may underlie its apparent influence on multiple biological processes in the newborn is an ability to alter the developmental expression of CYP enzyme activity.

The CYPs are a superfamily of heme-containing phase I enzymes responsible for biotransformation of a large number of endogenous substrates and a significant number of com-

Received March 16, 2006; accepted July 31, 2006.

Correspondence: Michael J. Blake, M.D., Ph.D., Division of Pediatric Pharmacology and Medical Toxicology, The Children's Mercy Hospitals and Clinics, 2401 Gillham Road, KS City, MO 64108; e-mail: mjblake@cmh.edu

Supported in part by grants 2 U01 HD31313-12 [Network of Pediatric Pharmacology Research Units (G.L.K.)] and 1 F32 HD043625-01 (M.J.B.), National Institute of Child Health and Human Development, Bethesda, MD.

DOI: 10.1203/01.pdr.0000245909.74166.00 monly used pharmacologic agents (5). They play a critical role in maintaining homeostasis by ridding the body of byproducts of cellular metabolism and waste products derived from dietary or other exogenous sources. Exposure to drugs, environmental toxins, and even dietary components has been shown to dramatically alter CYP expression and functional activity in adults (6). Moreover, the expression of members of several CYP families is dependent on development (7). Generally, CYP expression and activity are low in fetal tissue, with increasing activity apparently triggered by postnatal exposures or events (8).

Activities of specific CYP enzymes can be assessed in vivo by administration of specific probe substrates followed by quantifying the amount of parent compound and metabolites present in serum or urine. Oral caffeine administration is commonly used as a pharmacologic probe to assess CYP1A2 activity in vivo (9). Previous studies indicate that caffeine metabolism develops gradually during the first year of life (10) with its elimination half-life being prolonged in breastfed infants compared with formula-fed infants (11). These results were interpreted to suggest that breast milk may contain an inhibitor of caffeine metabolism. In light of more recent studies in adults showing that CYP1A2 activity can be induced by dietary constituents (12), it may be more likely that component(s) of commercial infant formulas increase CYP1A2 activity in developing infants. Less is known about the effects of infant diet on the development of other CYP enzymes. However, expression and induction of CYP3A and CYP2B enzymes have been shown to be increased by diet in a rodent model (13).

Based on these preliminary data from both animal and human studies, we hypothesized that infant feeding modality may significantly alter the developmental profile of specific CYP enzymes. The objective of this study was to assess differences in the development of functional CYP activity between healthy breast-fed and formula-fed infants administered caffeine and DM as metabolic probes.

Abbreviations: CYP, cytochrome P-450; DM, dextromethorphan; DX, dextrorphan; frDX, molar fractional recovery of DM; fr3HM, molar fractional recovery of 3HM; 3HM, 3-hydroxymorphinan 


\section{MATERIALS AND METHODS}

Participants and study protocol. Newborns recruited from well-baby nurseries at two local hospitals were seen at $2 \mathrm{wk}$ and then again at 1, 2, 3, 4, and 6 mo postnatal age. At their first visit, informed consent was obtained, newborn screens were checked, a birth history and physical examination were performed, and blood samples were obtained for laboratory tests (complete blood count, liver function tests, electrolyte panel). Any abnormal newborn screen, laboratory test, or physical finding prompted immediate referral for appropriate evaluation and treatment with exclusion or removal of an infant from the study.

At all visits, an interim history of infant diet, medication use, and exposure to environmental xenobiotics (e.g. cigarette/tobacco smoke) was obtained. Mothers of breast-fed infants were also queried about their dietary habits and asked to abstain from caffeinated food or beverages and medications containing DM $24 \mathrm{~h}$ before a study visit and during sampling. Healthy infants were administered oral caffeine citrate $(7 \mathrm{mg} / \mathrm{kg}$; prepared by dissolving the powder in sterile water and mixing the solution 2:1 with cherry syrup) and DM $(0.3 \mathrm{mg} / \mathrm{kg}$; supplied as Robitussin Pediatric Cough, Whitehall Robbins Healthcare, Madison, NJ). They were then fitted with preweighed fiber-based diapers (Tushies, Tender Care) for urine collection over the next $24 \mathrm{~h}$. At the 2 -wk and 2-, 4-, and 6-mo visits, approximately $0.3 \mathrm{~mL}$ of blood was drawn by heelstick $2 \mathrm{~h}$ after drug administration and again at their return visit the following day (approximately $24 \mathrm{~h}$ ). Blood samples were sent to the Children's Mercy Hospital Clinical Laboratory for determination of caffeine levels.

Investigators had no influence on the choice of feeding modality or choice of formula. All procedures involving human subjects were conducted according to the Declaration of Helsinki principles and received approval from the institutional review boards of the participating hospitals.

Serum caffeine analysis. Serum caffeine levels were determined by HPLC using a method that conforms to the guidelines of the College of American Pathologists Clinical Laboratory Improvement Act. Serum $(100 \mu \mathrm{L})$ was added to $500 \mu \mathrm{L}$ of an aqueous-based internal standard ( $\beta$-hydroxytheophylline; $5 \mu \mathrm{g} / \mathrm{mL}$; Sigma Chemical Co., St. Louis, MO) and passed over a solid-phase cartridge (Accubond C-18; Agilant, Palo Alto, CA). Eluted samples were injected onto the HPLC system (Hewlett Packard 1050). Analytes of interest were separated on a C18 column $(4.6 \times 250 \mathrm{~mm}, 5 \mu \mathrm{m})$ using a mobile phase of methanol:deionized water (10:90) at a flow rate of $3 \mathrm{~mL} / \mathrm{min}$. Eluate was monitored by UV detection at $275 \mathrm{~nm}$ with caffeine concentration determined against calibration standards that were run daily. The method is linear between $0.5-30 \mathrm{mg} / \mathrm{mL}$ with an intra- and interassay coefficient of variation for all standards that averaged $5 \%$.

Urine collection. Study diapers were weighed, and fecal matter was removed by separating the inner lining of the diaper from the absorptive matrix. Urine was expressed by manual extrusion of the absorptive matrix using a $60-\mathrm{mL}$ syringe. Ascorbic acid was added to urine samples (final concentration of $10 \mathrm{mg} / \mathrm{mL}$ ) in an attempt to stabilize amino-6-formylamino3-methyluracil (AFMU). All samples were stored at $-70^{\circ} \mathrm{C}$ before assay in the Pediatric Pharmacology Research Unit (PPRU) core laboratory of the Children's Mercy Hospitals and Clinics.

Urine caffeine and metabolites. Weighted, pooled urine samples from the 24-h sampling period were adjusted to a $\mathrm{pH}$ between 4 and 5 with sodium acetate $(250 \mathrm{mmol} / \mathrm{L})$ and filtered through a $0.22-\mu \mathrm{m}$ nylon centrifuge tube filter (Spin-X; Costar) before analysis. Caffeine and six of its major metabolites were determined in urine samples by reverse-phase HPLC with UV detection (Hewlett Packard HP1100; San Fernando, CA). Samples (20 $\mu \mathrm{L}$ ) were injected onto the HPLC system with analytes of interest eluted on a C18 column $(4.6 \times 250 \mathrm{~mm}, 5 \mu \mathrm{m}$; Aqua-C18; Phenomenex; Torrance, CA) using a mobile phase of methanol:0.1\% acetic acid (85:15) pumped at a flow rate of $1 \mathrm{~mL} / \mathrm{min}$. Eluates were monitored at 275 and $290 \mathrm{~nm}$ with caffeine and metabolite concentrations determined by use of peak height ratios against calibration standards comprising a 7-point standard curve. Standard curves for all metabolites were linear over a range of $6-200 \mu \mathrm{mol} / \mathrm{L}\left(r^{2}>0.99\right)$. The coefficient of variation for all standards was $<10 \%$ except for the limit of quantification (LOQ), which was consistently $<20 \%$. Initially, samples were evaluated for the presence of 3,7-dimethylxanthine, 3,7-dimethylurate, 7-methlyxanthine, and 3-methylurate in addition to those caffeine metabolites reported in this study (Fig. 1). However, none of these compounds were consistently present above the limits of quantitation in our samples. Molar fractions of caffeine and its measured metabolites were defined as (metabolite or caffeine) $/(137 \mathrm{X}+1 \mathrm{X}+1 \mathrm{U}+17 \mathrm{X}+17 \mathrm{U}+13 \mathrm{X}+3 \mathrm{X}$ ) (see Fig. 1 legend for abbreviations).

Urine DM and metabolites. Pooled urine samples were adjusted to a $\mathrm{pH}$ between 4 and 5 with sodium acetate $(50 \mathrm{mmol} / \mathrm{L} ; \mathrm{pH}=3)$. Samples were deconjugated by the addition of $10 \mu \mathrm{L}$ of $\beta$-glucuronidase $(100,400 \mathrm{U} / \mathrm{mL}$; Sigma Chemical Co., St. Louis, MO) to each $\mathrm{ml}$ of urine and incubated

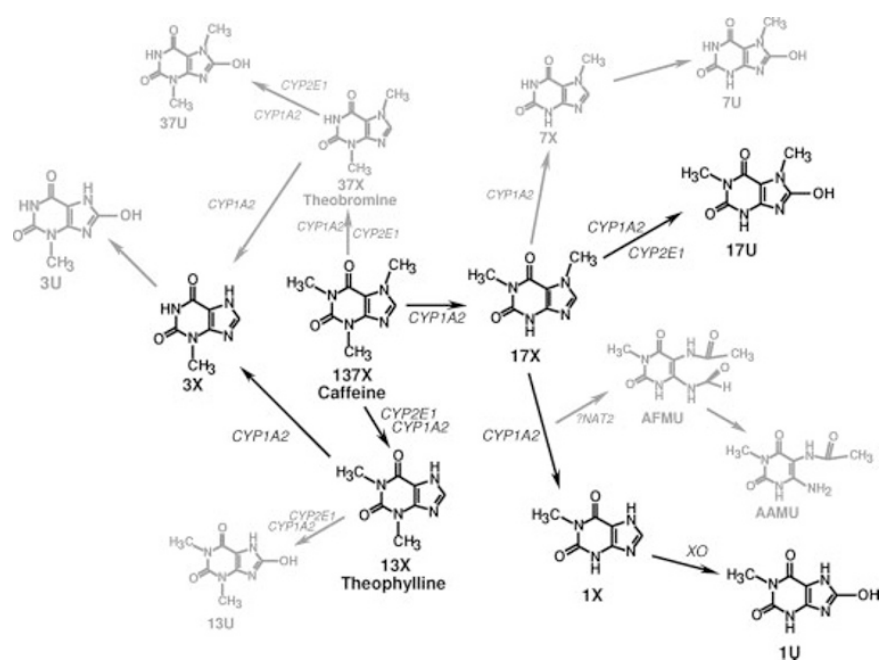

Figure 1. Proposed pathways of methylxanthine metabolism. 137X: 1,3,7trimethylxanthine (caffeine); 17X: 1,7-dimethylxanthine; 17U: 1,7dimethyluracil; 1X: 1-methylxanthine; 1U: 1-methyluracil; 7X: 7-methylxanthine; 7U: 7-methyluracil; 13X: 1,3-dimethylxanthine (theophylline); 13U: 1,3-dimethyluracil; 3X: 3-methylxanthine; 3X: 3-methyluracil; 37X: 3,7dimethylxanthine (theobromine); 37X: 3,7-dimethyluracil; AFMU: 5-acetylamino-6-formylamino-3-methyluracil; AAMU: 5-acetylamino-6amino-3-methyluracil. NAT2: $N$-acetyltransferase; CYP1A2: cytochrome P-450 1A2; CTP2E1: cytochrome P-450 2E1. Only metabolites in bold were consistently present above the limit of detection in urine of infants in this study.

overnight $(18 \mathrm{~h})$ at $37^{\circ} \mathrm{C}$. Levallorphan tartrate $(10 \mu \mathrm{L} ; 50 \mu \mathrm{g} / \mathrm{mL})$ was added to each milliliter of deconjugated sample (internal standard), followed by filtration using a $0.22-\mu \mathrm{m}$ nylon centrifuge tube filter (Spin-X; Costar). DM and three of its major metabolites were determined by reverse-phase HPLC (Hewlett Packard HP1100; San Fernando, CA). Samples $(20 \mu \mathrm{L})$ were injected onto the HPLC system with analytes of interest eluted on a C18 column $(4.6 \times 150 \mathrm{~mm}, 5 \mu \mathrm{m}$; Nova Phenyl; Phenomenex; Torrance, CA) and eluted with a mobile phase consisting of $10 \mathrm{mmol} / \mathrm{L}$ sodium acetate $(\mathrm{pH}=4)$ : acetonitrile $(23: 77)$ pumped at a flow rate of $1 \mathrm{~mL} / \mathrm{min}$. Eluates were monitored by fluorescence detection (235 and $310 \mathrm{~nm}$ ) with DM and metabolite concentrations determined by peak height ratio comparison to a 7-point standard curve prepared before each run. Standard curves for DM, dextrorphan (DX), 3-hydroxymorphinan (3HM), and 3-methoxymorphinan $(3 \mathrm{MM})$ were linear over a range of $0.1-10 \mu \mathrm{mol} / \mathrm{L}\left(r^{2}>0.99\right)$. The coefficient of variation for all standards was consistently $<10 \%$. DM and 3MM levels were consistently below the limit of detection and were not included in subsequent analyses. The molar fractional recovery of DX (frDX) and 3HM (fr3HM) was defined as DX/(DX + 3HM) and 3HM/(DX + 3HM), respectively. The molar ratio of 3HM/DX was used as to estimate CYP3A4 activity.

Data analysis. The apparent elimination rate constant was calculated from serum caffeine concentrations using the following equation: $\mathrm{k}_{\mathrm{e}}=\left(\ln \mathrm{C}_{1}-\ln \mathrm{C}_{2}\right) /$ $\left(t_{1}-t_{2}\right)$. Blood sample collection times were recorded and used for this calculation. The fractional molar recoveries of caffeine and its metabolites were determined from pooled urine samples collected over a 24-h postdose period. Due to the collection of multiple samples from the same individual over time, a linear, mixed model analysis with random intercepts was determined to be the most appropriate statistic to concomitantly evaluate changes due independently to both age and diet (14). For these analyses, cumulative exposure (in weeks) to either formula or breast milk was determined at each study visit. Infants reported to have received any combination of formula and breast milk at a study visit were considered to have received a diet exposure equivalent to one half of the interval between visits. These cumulative exposure estimates allowed comparisons to be made between diets over the 6-mo study period and account for those infants who were initially breast-fed and then switched to formula during the study. This analysis, along with analysis of variance with simple effects (Bonferroni) and linear regression analyses were performed using SPSS software. The level of significance accepted for all statistical analyses was $\alpha=0.05$. 
Table 1. Participant demographics

\begin{tabular}{lc}
\hline \multicolumn{1}{c}{ Demographics } \\
\hline No. of subjects enrolled & 48 \\
Failed screen/dropouts & 10 \\
Completed $>$ 5 study visits & 35 \\
Sex & 19 \\
Male & 19 \\
Female & \\
Race & 14 \\
African American & 17 \\
White & 3 \\
Hispanic & 4 \\
Other & \\
Gestational age at birth, wk & $34-43$ \\
Range
\end{tabular}

Consent was obtained from 48 participants. Ten failed initial screening or dropped out before completing a study visit. Thirty-eight infants completed two or more study visits and were included in the data analysis.

\section{RESULTS}

Of 48 infants enrolled in the study, 38 completed two or more study visits and were included in the data analysis (Table 1). A reported history of smoking had no effect on caffeine elimination in our patient population (data not shown). Table 2 details the numbers of infants receiving a specific diet at each study visit. Infants categorized as either breast-fed or formula fed received $>95 \%$ of their intake exclusively from these sources. Infants receiving a combination of breast milk or formula during an interim period were categorized as supplemented. Forty-four percent of infants seen at 2 wk postnatal age were exclusively breast-fed. Consistent with current trends (15), only $23 \%$ of infants were exclusively breast-fed by their 6-mo visit.

The relationship between postnatal age and the caffeine elimination rate constant $\left(\mathrm{k}_{\mathrm{e}}\right)$ in all study participants is depicted in Figure 2. Serum caffeine elimination was very low at 2 wk of age followed by a progressive increase in rate over the first 6 mo of life. Regression analysis of these data revealed a positive, linear correlation between $\mathrm{k}_{\mathrm{e}}$ and postnatal age $\left(r^{2}=\right.$ 0.469; $p<0.001)$.

To address whether developmental changes in caffeine elimination occur before birth, a regression analysis of $\mathrm{k}_{\mathrm{e}}$ at the first study visit versus postconceptional age was performed. The postconceptional age of infants at their first study visit (2-3 wk postnatal age) ranged between 36 and 43 wk.

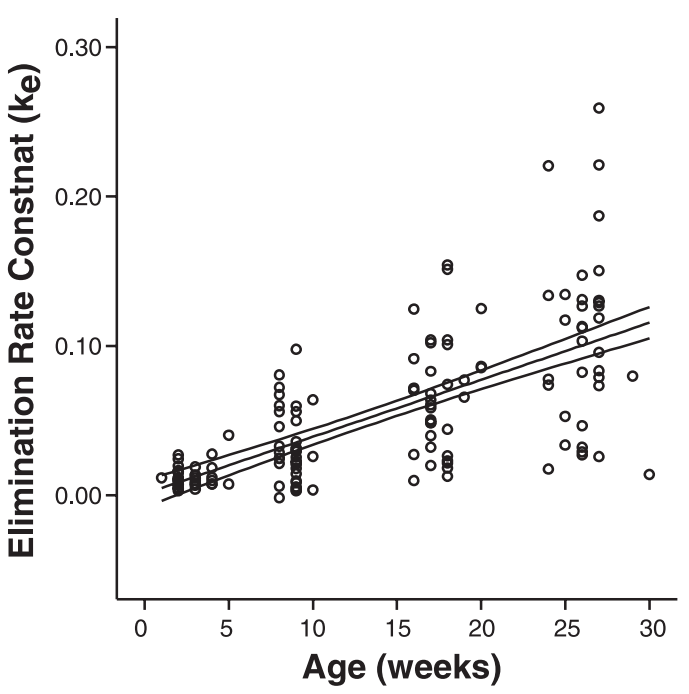

Figure 2. Relationship between caffeine $\mathrm{k}_{\mathrm{e}}$ and postnatal age. Lines represent linear regression $\pm 95 \%$ confidence intervals. Regression analysis indicated a significant positive linear correlation between caffeine elimination and age $\left(r^{2}=0.469 ; p<0.01\right)$. The numbers of infants included in the analysis at each study visit are as indicated in Table 2.

Although the postconceptional age of the infants spanned almost 2 mo, no significant relationship was identified with $\mathrm{k}_{\mathrm{e}}$.

Figure 3 summarizes the relationship between serum caffeine $\mathrm{k}_{\mathrm{e}}$ and postnatal age stratified by diet. Caffeine elimination increased with age in infants exposed to all diets. At 2-3 and 4-6 wk of age, there were no significant differences in $\mathrm{k}_{\mathrm{e}}$ between diets. At all remaining study visits, caffeine elimination was markedly and progressively greater in formula-fed infants than in infants exclusively breast-fed $(p<0.01)$. Caffeine elimination in supplemented infants was never statistically different from either formula or breast-fed infants, likely reflecting the relatively small number of babies in this diet group (Table 2). However, their average values were consistently comparable to breast-fed infants.

The relative proportion of urinary caffeine metabolites in all infants, regardless of diet, is displayed in Figure 4. Urine from infants 2-3 or 4-6 wk of age contained predominantly unmetabolized caffeine (137X). At subsequent visits, the relative proportion of all caffeine metabolites increased as the proportion of unmetabolized caffeine decreased. 1-Methylurate was present earliest (2-3 wk) and its relative proportion increased

Table 2. Diet summary

\begin{tabular}{|c|c|c|c|c|c|c|c|}
\hline & \multicolumn{6}{|c|}{ Diet summary } & \multirow[b]{2}{*}{ Total } \\
\hline & Breast-fed & $\begin{array}{l}\text { Breast-fed } \\
\text { and solids }\end{array}$ & Supplemented & $\begin{array}{l}\text { Supplemented } \\
\text { and solids }\end{array}$ & Formula-fed & $\begin{array}{c}\text { Formula-fed } \\
\text { and solids }\end{array}$ & \\
\hline $2 \mathrm{wk}$ & 15 & 0 & 7 & 0 & 12 & 0 & 34 \\
\hline $4 \mathrm{wk}$ & 13 & 0 & 8 & 0 & 14 & 2 & 37 \\
\hline $8 \mathrm{wk}$ & 12 & 0 & 4 & 1 & 16 & 4 & 37 \\
\hline $12 \mathrm{wk}$ & 9 & 0 & 4 & 1 & 18 & 5 & 37 \\
\hline $16 \mathrm{wk}$ & 9 & 0 & 1 & 1 & 17 & 7 & 35 \\
\hline $24 \mathrm{wk}$ & 6 & 2 & 1 & 1 & 9 & 16 & 35 \\
\hline
\end{tabular}

At each study visit, an interim diet history was obtained from participant mothers. Infants were considered breast-fed or formula fed if they received $>95 \%$

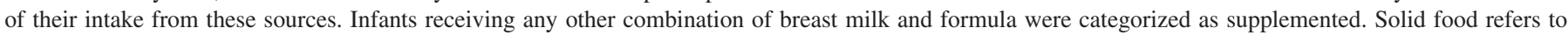
infant cereal, infant pureed jar food, or table food. 


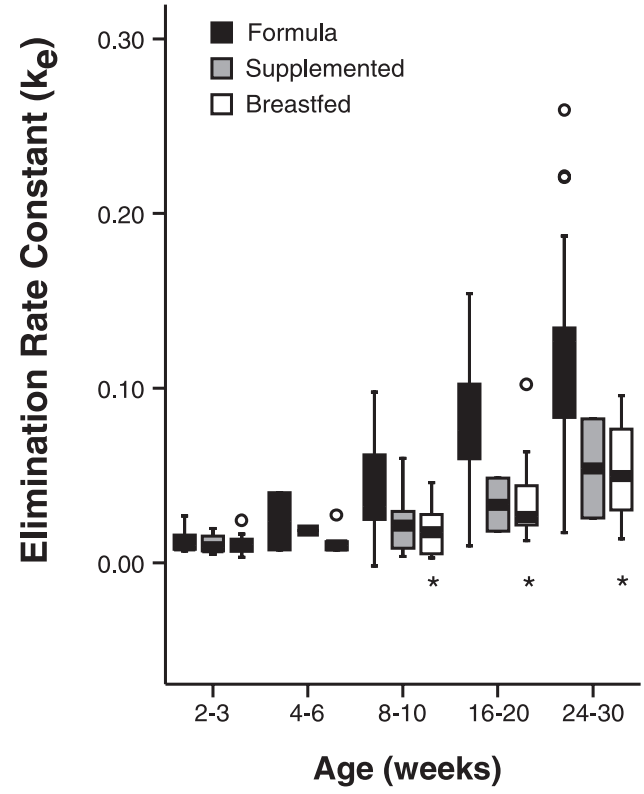

Figure 3. Effect of diet on caffeine $\mathrm{k}_{\mathrm{e}}$ with age. Boxes represent the interquartile range; horizontal bars in the boxes represent median values; whiskers represent the 10th-90th percentiles; circles represent values between 1.5 and 3 boxes from the interquartile range. An asterisk indicates the mean $\mathrm{k}_{\mathrm{e}}$ is significantly different from formula-fed infants $(p<0.01)$. The numbers of infants receiving a specific diet at each study visit are as indicated in Table 2.

most rapidly. By 6 mo of age, it was the most prevalent form of caffeine recovered from urine samples. 3-Demethylated compounds [1,7-dimethylxanthine (17X); 1,7-dimethylurate $(17 \mathrm{U})]$ were the next most prevalent caffeine metabolites and showed a similar relative increase with age. 7-Demethylated compounds [1,3-dimethylxanthine (13X); 3-methylxanthine $(3 \mathrm{X})$ ] showed a more variable age-related progression and were the least abundant of the caffeine metabolites.

The molar fraction of caffeine and its metabolites were stratified according to diet (Fig. 5). Infants receiving a supplemented diet were not included in this analysis due to their relatively small numbers. The proportion of unmetabolized caffeine was similar in breast-fed and formula-fed infants at their first three visits (Fig. 5A), but was consistently smaller in formula-fed infants at subsequent visits. Linear mixed model analysis indicated a significant decrease in the proportion of

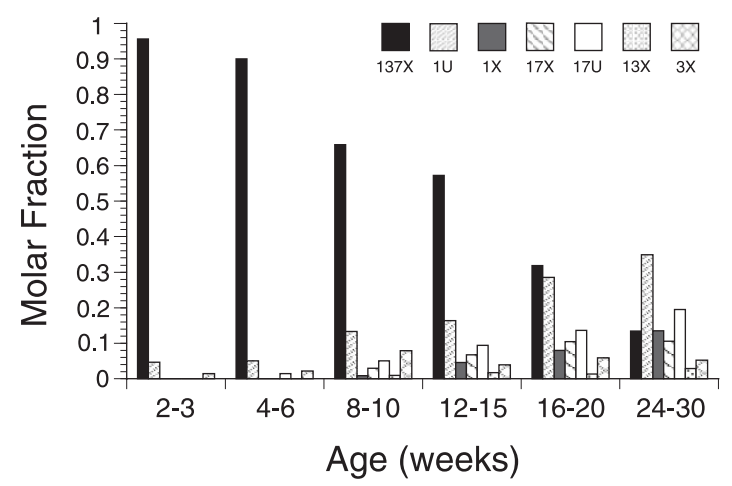

Figure 4. Fractional molar recovery of caffeine and six of its metabolites in urine samples collected during study visits. Abbreviations are as described in Figure 1. unmetabolized caffeine in urine with age $(y=-0.028 x+1$; $p<0.001)$ and a further decrease due to formula exposure beyond that expected from age alone $(y=-0.010 x+1$; $p<0.01)$. Conversely, the molar fractions of $17 \mathrm{X}$ and $1 \mathrm{X}$ were greater in formula-fed infants than breast-fed infants at all visits beyond $4-6 \mathrm{wk}$ of age (Fig. $5 B$ and $C$ ). Linear mixed model analyses showed a significant increase in the molar ratio of both $17 \mathrm{X}$ and $1 \mathrm{X}$ with age and an independent increase in these ratios associated with formula exposure. The molar fraction of the remaining caffeine metabolites were more variable and showed no consistent correlation with infant diet.

The effect of age and diet on the metabolism of DM is depicted in Figure 6. DX constituted almost 90\% of DM metabolites at the first study visit (Fig. 6A). With increasing postnatal age, the frDX decreased to approximately 50\%, whereas the fr3HM increased from just over $10 \%$ at the first study visit (2-3 wks) to approximately 50\% of total metabolites by $6 \mathrm{mo}$ of age. The increasing prevalence of $3 \mathrm{HM}$ at the expense of DX reflects an apparent increase in DM $N$ demethylation during this developmental time period (Fig. 7). Ratios of urinary 3HM/DX have been shown to correlate well with midazolam clearance in adults, suggesting this ratio provides a surrogate for CYP3A enzyme activity in vivo (16). The 3HM/DX molar ratio in urine from infants in this study were stratified by diet and plotted as a function of age (Fig. $6 B)$. Regression analysis indicated a significant increase in 3HM/DX with age in both formula-fed $\left(r^{2}=0.192 ; p<\right.$ $0.001)$ and breast-fed $\left(r^{2}=0.455 ; p<0.001\right)$ infants. Linear mixed model analyses indicated that the rate of increase in the molar ratio of 3-HM/DX with age occurred faster in formulafed infants [0.012/wk 95\% confidence interval (CI): 0.009$0.014]$ than in breast-fed infants $(0.005 /$ wk $95 \%$ CI: $0.001-$ $0.008)(p<0.001)$.

\section{DISCUSSION}

Consistent with previously published results (10), we observed an increase in caffeine elimination over the first 6 mo of life. By 2 mo of age, the average caffeine $\mathrm{k}_{\mathrm{e}}$ was already greater than that observed in 2-wk-old infants, indicating that a measurable increase in caffeine metabolism occurs as early as $1.5 \mathrm{mo}$ of postnatal age. Although postconceptional age spanned almost 2 mo, no significant relationship with caffeine $\mathrm{k}_{\mathrm{e}}$ was observed at the 2-wk visit. Furthermore, negligible amounts of caffeine metabolites were detected in urine at their first visit (Fig. 4). Collectively, these results corroborate previous data $(10,17)$ that suggest that there is minimal ability to metabolize caffeine before birth. Rather, it appears that postnatal exposures or events are required before the capacity to metabolize caffeine begins to mature.

Results of this study provide evidence that an infant's dietary exposure plays a significant role in the development of caffeine metabolism. The ability to metabolize caffeine developed faster in infants consuming formula than those fed breast milk. Collective results suggest that more rapid caffeine elimination in formula-fed infants occurs as a consequence of increased conversion of caffeine to its 3-demethylated (17X) 

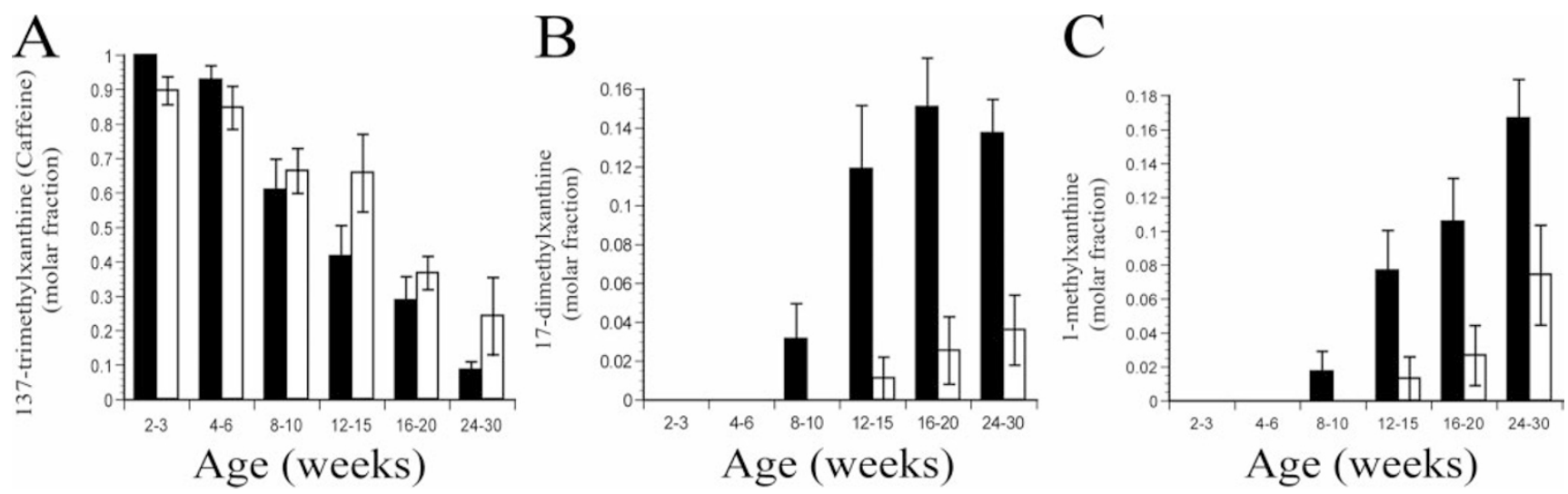

Figure 5. Effect of infant diet on the fractional molar recovery: 1,3,7-trimethylxanthine (caffeine) (A); 17X: 1,7-dimethylxanthine (B); and 1-methylxanthine (C). Bars represent average fractional molar recovery \pm standard error of the mean (SEM). Linear mixed model analysis indicated that consumption of infant formula corresponded to faster metabolism of caffeine reflected by reduced amounts of unmetabolized caffeine and greater amounts of $17 \mathrm{X}$ and $1 \mathrm{X}$ in urine samples $(p<0.001)$. The number of breast-fed (open columns) and formula-fed (filled columns) infants in each group are as indicated in Table 2. Supplemented infants were not included in these analyses.

and 3,7-demethlyated (1X) metabolites. Linear mixed model analyses indicated that formula feeding increased the proportion of these metabolites independent of the effects of age alone. Whether this effect is due to the presence of an inducer in formula or an inhibitory factor in breast milk remains to be elucidated.

Levels of urinary caffeine and its metabolites have been used in various combinations to produce molar ratios proposed as surrogate markers of CYP1A2 activity in adults (9). The small amounts of caffeine metabolites in our very young infants precluded the use of these ratios in our study. In an attempt to determine a urinary caffeine metabolite ratio that best predicted caffeine elimination in our infant participants, linear regression analyses were performed between several ratios of urinary caffeine metabolites and the serum $\mathrm{k}_{\mathrm{e}}$. A strong correlation was observed between the urinary molar ratio of $(17 \mathrm{X}+1 \mathrm{X}) /$ caffeine and caffeine $\mathrm{k}_{\mathrm{e}}\left(r^{2}=0.57 ; p<\right.$ $0.001)$. When postnatal age was added as a covariant to this analysis $\left\{\mathrm{k}_{\mathrm{e}}=0.016[(17 \mathrm{X}+1 \mathrm{X}) /\right.$ caffeine $\left.)\right]+0.002$ (age in
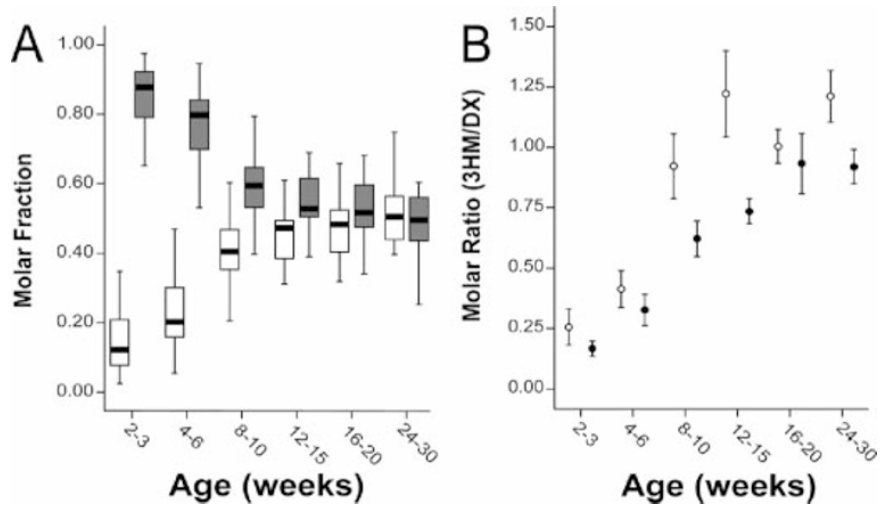

Figure 6. Effect of age on DM metabolism. (A) Box plots of fr3HM (open columns) and frDX (filled columns). Box plots are as defined in Figure. 3. (B) Mean \pm SEM of the molar ratio 3HM/DX in formula-fed (open circles) and breast-fed (filled circles) infants. Linear mixed model analysis indicated a faster development of CYP3A4 activity as assessed by 3HM/DX in formulafed infants than in those receiving breast milk $(p<0.001)$. The numbers of breast-fed and formula-fed infants in each group are as indicated in Table 2. Supplemented infants were not included in these analyses. weeks $)+0.009\}$, the predictive value increased further $\left(r^{2}=\right.$ $0.65 ; p<0.001)$. The predictive value of this equation compares favorably to correlations between caffeine elimination and urinary metabolites in adults (18).

The 3-demethylation of caffeine to $17 \mathrm{X}$ and its subsequent conversion to $1 \mathrm{X}$ has been shown to be catalyzed predominantly by CYP1A2 (19) (Fig. 1). Moreover, previous studies employing animal models and in vitro studies using human liver microsomes suggest that CYP1A2 activity is actively maturing during the first 6 mo of life $(10,17)$. Thus, increasing amounts of $17 \mathrm{X}$ (and 1X) with age observed in this study likely reflect maturation of CYP1A2 activity. Indeed, accelerated formation of these caffeine metabolites in formula-fed infants may occur as a result of maturation of CYP1A2 enzyme activity that is augmented by this dietary exposure.

Similarly, the DM metabolite ratio (3HM/DX) used in this study may reflect maturation of CYP3A4 activity. Figure 7 summarizes the known biotransformation pathway of DM $(20,21)$. CYP2D6 activity appears to be present by 2 wk of age as very little $(<2 \%)$ DM was detected in urine of 2-3-wk-old infants, whereas DX constituted almost $90 \%$ of recovered DM
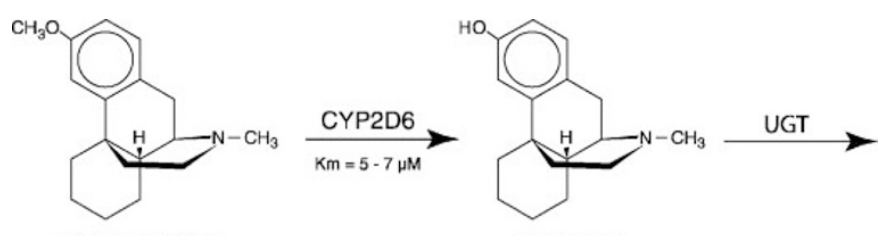

Dextromethorphan

Dextrorphan
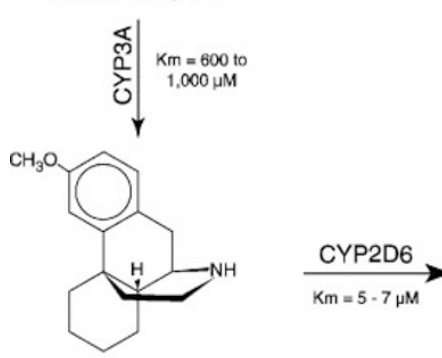

$\varangle$

$\mathrm{Km}=1,600$ to

u
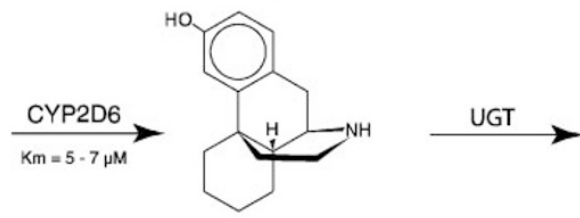

3-Methoxymorphinan

3-Hydroxymorphinan

Figure 7. Proposed pathways of DM metabolism. 
metabolites. As the infants aged, the relative proportion of DX decreased with a corresponding increase in the proportion of $3 \mathrm{HM}$. The increase in $3 \mathrm{HM}$ with age likely reflects an increased $\mathrm{N}$-demethylation of DM to 3-methoxymorphinan followed by rapid conversion to 3HM (i.e. 3-methoxymorphinan was consistently below the level of detection) or possibly a direct conversion of DX to 3HM. Since conversion of DM to 3-methoxymorphinan has been shown to be catalyzed by CYP3A4 (20) and to a lesser extent by CYP2B6 (21) in adults, the increase in $3 \mathrm{HM}$ with age may reflect the developmental maturation of one or both of these enzymes. Ratios of urinary 3HM/DX have been shown to provide an acceptable surrogate for CYP3A enzyme activity in vivo (16). We observed higher 3HM/DX ratios in formula-fed infants than in breast-fed infants (Fig. $6 \mathrm{~B}$ ) at all sampling periods. Thus, similar to CYP1A2, maturation of CYP3A4 enzyme activity may also be accelerated in formula-fed infants.

Molecular mechanisms regulating the expression of CYP1A2 and CYP3A have been described. CYP1A2 expression can be induced by activation of the aryl hydrocarbon receptor (AhR) and subsequent increased transcription of the CYP1A2 gene (22). CYP3A4 expression can be induced by activation of either the pregnane $\times$ receptor $(\mathrm{PXR})$ or constitutive androstane receptor (CAR), which forms heterodimers with the retinoic acid receptor (RXR) before binding to response elements on the CYP3A4 gene (23). The ability of xenobiotics to increase CYP1A2 or CYP3A4 levels and activity in adults appears to work through these mechanisms. Similarly, there may be specific ligand(s) present in infant formula and not in human breast milk that can activate these nuclear receptors, ultimately resulting in increased enzyme activity. In fact, a recent report provides compelling data that infant formula contains AhR ligand(s) that cause a marked up-regulation of CYP1A1/1A2 mRNA and protein in a human hepatoma cell line (24). CYP3A4 mRNA was induced to a lesser extent by both breast milk and formula in this study, but did not appear to involve substantial activation of PXR.

Alternatively, formula may affect maturational events independent of known induction mechanisms. Whereas increased expression in adults occurs by activity of these transcription factors, exposure to infant formula may increase the rate of acquisition of basal levels of expression. Thus, it may not be that infant diet is inducing CYP1A2 or CYP3A4 activity per se. Rather, it may accelerate the maturational expression of these enzymes independent of induction. Whether similar regulatory mechanisms control both maturational processes and induction has not been established.

The consequence of increased CYP1A2 and CYP3A4 activity following exposure to infant formula remains a salient question. Our results do not espouse an advantage to either diet, but rather highlight differences. The short-term consequences of these differences are readily apparent in that altered infant metabolism of drugs by dietary components may affect their disposition and as a result their pharmacodynamics or toxicity. In the long term, accelerated development of phase I enzyme activity by infant formula may alter exposure to modified xenobiotics and endogenous metabolites from a very early age. Chronic exposure to these potentially reactive me- tabolites coupled with genetic predisposition may contribute to differences in susceptibility to specific disease processes (neoplasms, autoimmune dysfunction). Accordingly, caffeine itself has been reported to inhibit topoisomerase I (25) and the carcinogenic potential of some environmental toxins is thought to be mediated by altered CYP1A expression (26).

Neonates and young infants are in a dynamic state of maturation that apparently has a dramatic influence on the regulation of the genes responsible for the production and/or activation of drug metabolizing enzymes. Our results provide further insight into the developmental regulation of CYP functional activity and, in particular, the potential for dietary constituents to modulate "normal" developmental expression. Future studies that identify the specific constituent(s) of an infant's diet that modulates CYP activity may help define the cellular and genetic mechanisms responsible for diet-CYP interactions and their physiologic significance.

Acknowledgments. The authors gratefully acknowledge the assistance of Debra Taylor, R.N., for her aid in patient management, Lin Tao for his technical assistance, and Dr. Stephen Simon for his direction and review of the statistical analysis.

\section{REFERENCES}

1. Goldman AS 2000 Modulation of the gastrointestinal tract of infants by human milk. Interfaces and interactions. An evolutionary perspective. J Nutr 130:426S-431S

2. Kwan ML, Buffler PA, Abrams B, Kiley VA 2004 Breastfeeding and the risk of childhood leukemia: a meta-analysis. Public Health Rep 119:521-535

3. Oddy WH 2004 A review of the effects of breastfeeding on respiratory infections, atopy, and childhood asthma. J Asthma 41:605-621

4. Morrow AL, Ruiz-Palacios GM, Jiang X, Newburg DS 2005 Human-milk glycans that inhibit pathogen binding protect breast-feeding infants against infectious diarrhea. J Nutr 135:1304-1307

5. Nelson DR, Koymans L, Kamataki T, Stegeman JJ, Feyereisen R, Waxman DJ, Waterman MR, Gotoh O, Coon MJ, Estabrook RW, Gunsalus IC, Nebert DW 1996 P450 superfamily: update on new sequences, gene mapping, accession numbers and nomenclature. Pharmacogenetics 6:1-42

6. Lin JH, Lu AY 2001 Inter-individual variability in inhibition and induction of cytochrome P450 enzymes. Annu Rev Pharmacol Toxicol 41:535-567

7. Leeder JS 2001 Pharmacogenetics and pharmacogenomics. Pediatr Clin North Am 48:765-781

8. Blake MJ, Castro L, Leeder JS, Kearns GL 2005 Ontogeny of drug metabolizing enzymes in the neonate. Semin Fetal Neonatal Med 10:123-138

9. Nordmark A, Lundgren S, Cnattingius S, Rane A 1999 Dietary caffeine as a probe agent for assessment of cytochrome P4501A2 activity in random urine samples. Br J Clin Pharmacol 47:397-402

10. Aldridge A, Aranda JV, Neims AH 1979 Caffeine metabolism in the newborn. Clin Pharmacol Ther 25:447-453

11. Le Guennec J-C, Billon B 1987 Delay in caffeine elimination in breast-fed infants. Pediatrics 79:264-268

12. Fontana RJ, Lown KS, Paine MF, Fortlage L, Santella RM, Felton JS, Knize MG Greenberg A, Watkins PB 1999 Effects of a chargrilled meat diet on expression of CYP3A, CYP1A, and P-glycoprotein levels in healthy volunteers. Gastroenterology 117:89-98

13. Ronis MJ, Rowlands JC, Hakkak R, Badger TM 1999 Altered expression and glucocorticoid-inducibility of hepatic CYP3A and CYP2B enzymes in male rats fed diets containing soy protein isolate. J Nutr 129:1958-1965

14. McColloch CC, Seale SR 2001 Generalized, Linear, and Mixed Models. John Wiley \& Sons, New York

15. Ahluwalia IB, Morrow B, Hsia J, Grummer-Strawn LM 2003 Who is breast-feeding? Recent trends from the pregnancy risk assessment and monitoring system. J Pediatr 142:486-491

16. Kawashima Y, Hagiwara M, Inoue Y, Someya T 2002 Evaluation of dextromethorphan $N$-demethylation activity as a biomarker for cytochrome P450 3A activity in man. Pharmacol Toxicol 90:82-88

17. Aranda JV, Scalais E, Papageorgiou A, Beharry K 1984 Ontogeny of human caffeine and theophylline metabolism. Dev Pharmacol Ther 7:18-25

18. Denaro CP, Wilson M, Jacob P 3rd, Benowitz NL 1996 Validation of urine caffeine metabolite ratios with use of stable isotope-labeled caffeine clearance. Clin Pharmacol Ther 59:284-296

19. Gu L, Gonzalez FJ, Kalow W, Tang BK 1992 Biotransformation of caffeine, paraxanthine, theobromine and theophylline by cDNA-expressed human CYP1A2 and CYP2E1. Pharmacogenetics 2:73-77 
20. Jacqz-Aigrain E, Cresteil T 1992 Cytochrome P450-dependent metabolism of dextromethorphan: fetal and adult studies. Dev Pharmacol Ther 18:161-168

21. Yu A, Haining RL 2001 Comparative contribution to dextromethrophan metabolism by cytochrome P450 isoforms in vitro: Can dextromethorphan be used as a dual probe for both CYP2D6 and CYP3A activities? Drug Metab Dispos 29:1514-1520

22. Nebert DW, Dalton TP, Okey AB, Gonzalez FJ 2004 Role of aryl hydrocarbon receptor-mediated induction of the CYP1 enzymes in environmental toxicity and cancer. J Biol Chem 279:23847-23850

23. Maglich JM, Stoltz CM, Goodwin B, Hawkins-Brown D, Moore JT, Kliewer SA 2002 Nuclear pregnane X receptor and constitutive androstane receptor regulate overlapping but distinct sets of genes involved in xenobiotic detoxification. Mol Pharmacol 62:638-646

24. Xu H, Rajesan R, Harper P, Kim RB, Lonnerdal B, Yang M, Uematsu S, Hutson J, Watson-Macdonell J, Ito S 2005 Induction of cytochrome P450 1A by cow milkbased formula: a comparative study between human milk and formula. $\mathrm{Br} \mathrm{J}$ Pharmacol 146:296-305

25. Tohda H, Zhao JH, Oikawa A 1992 A possible involvement of DNA topoisomerase I in "caffeine effect" after ultraviolet irradiation. Tohoku J Exp Med 168:129-132

26. Badawi AF, Cavalieri EL, Rogan EG 2000 Effect of chlorinated hydrocarbons on expression of cytochrome P450 1A1, 1A2 and 1B1 and 2- and 4-hydroxylation of 17beta-estradiol in female Sprague-Dawley rats. Carcinogenesis 21:1593-1599 